\title{
Modeling and Solving the Tactical Berth Allocation Problem
}

\author{
Giovanni Giallombardo* Luigi Moccia ${ }^{\dagger}$ \\ Matteo Salani $\ddagger \quad$ Ilaria Vacca $\ddagger \S$
}

March 12, 2009

Report TRANSP-OR 090312

Transport and Mobility Laboratory

Ecole Polytechnique Fédérale de Lausanne

transp-or.epfl.ch

\footnotetext{
*DEIS, Università della Calabria, Italy

†Istituto di Calcolo e Reti ad Alte Prestazioni, CNR, Italy

¥Transp-OR, Ecole Polytechnique Fédérale de Lausanne, Switzerland

${ }^{\S}$ Corresponding author: ilaria.vacca@epfl.ch
} 


\begin{abstract}
In this paper we integrate at the tactical level two decision problems arising in container terminals: the berth allocation problem, which consists of assigning and scheduling incoming ships to berthing positions, and the quay crane assignment problem, which assigns to incoming ships a certain QC profile (i.e. number of quay cranes per working shift). We present two formulations: a mixed integer quadratic program and a linearization which reduces to a mixed integer linear program. The objective function aims, on the one hand, to maximize the total value of chosen $\mathrm{QC}$ profiles and, on the other hand, to minimize the housekeeping costs generated by transshipment flows between ships. To solve the problem we developed a heuristic algorithm which combines tabu search methods and mathematical programming techniques. Computational results on instances based on real data are presented and compared to those obtained through a commercial solver.
\end{abstract}




\section{Introduction}

Maritime transportation has gained a crucial role in the exchange of goods between continents and containerization enforced this trend (UNCTAD, 2008). In order to cut down transportation costs, container traffic asks for ultra-large containerships and thus for terminals with facilities and technologies able to handle them (mega-terminals), and for a maritime transportation system which can reduce transportation costs. This system is known as hub and spoke: deep sea containerships (mother vessels) operate among a limited number of transhipment terminals ( $h u b s$ ), and smaller vessels (feeders) link the hubs with the other ports (spokes). The need for an optimal management of logistic activities at modern container terminals is well recognized. For an overview and classification of the various equipments and decision problems in such systems, see Vis and de Koster (2003), Steenken et al. (2004), Crainic and Kim (2007), Stahlbock and Voss (2008), and Monaco et al. (2009).

This paper deals with the Tactical Berth Allocation Problem (TBAP) with integrated quay crane assignment and quadratic yard costs in a transshipment container terminal. It is well known that the Operational Berth Allocation Problem, in the following referred to as BAP according to the convention commonly adopted in the literature, consists of assigning and scheduling ships to berthing positions along the quay, with the aim of minimizing ships' turnaround time. In particular, the usual BAP planning horizon covers just few days, say at most one week, due to the uncertainties of maritime traveling times. Here, our specific motivation in building a tactical version of BAP, is not simply the obvious one of considering a longer planning horizon, say at least one week up to several weeks, but mainly that of supporting decisions made by terminal managers in the negotiation process with shipping lines. The terminal and the shipping line negotiate the expected vessel handling time which depends upon the number of assigned Quay Cranes (QCs) along a time axis. We indicate as a QC profile the number of QCs available for the vessel at each time step of its handling time, and we explicitly model as decision variables the selection of QC profiles. Such integration enables terminal managers to 
evaluate how performance of the terminal change with the different service intensity offered/required to/by the shipping lines. Therefore, negotiation amounts to evaluate the impact on the performance of guaranteeing certain QC profiles to the shipping lines, and, as a consequence, the main operating resources involved in this process are the berths and the quay cranes. The last remark has provided strong motivation to build a model arising from the integration, at the tactical level, of BAP with the Quay Crane Assignment Problem. While this will be clarified later in the paper, as for the remainder of the section we focus on the main features of TBAP in comparison with BAP, assuming the reader be quite familiar with existing BAP formulations.

Basically, both the tactical and the operational problems deal with assigning and scheduling ships to berthing positions, i.e. deciding where and when the ships should moor. Both the TBAP and the BAP strives to balance terminal costs and service quality. However, as already noted, the different decisional levels and time frames induce different problems. In the TBAP service quality depends upon the negotiation between the terminal and the shipping lines regarding the terminal resources. A higher service quality occurs when the terminal can accommodate shipping lines requests in term of expected berthing times, and assigned quay cranes. In the BAP service quality is measured by adherence to a schedule, e.g. ideally zero waiting time to moor.

The TBAP, thanks to the longer planning horizon, can integrate terminal's costs in a more comprehensive way with respect to the BAP. In a transshipment terminal, containers arrive and depart by vessels while being temporarily stored in the yard. When unloading a vessel, the discharged containers must be allocated to yard positions close enough to the vessel berthing point in order to speed up the vessel handling. However, when the departure position of a container is far from its yard position, the container must be reallocated before the arrival of the outbound vessel. Therefore, the yard management deals with a dynamic allocation of containers through their duration-of-stay inside the terminal, see Moccia et al. (2009). The operational BAP considers this aspect penalizing mooring far from the most favorable berth with respect to the yard positions of out- 
bound containers. It should be noted that is the tactical berth allocation that determines the long-term favorite berth (home berth) for each vessel, thus inducing container flows inside the yard. In the BAP the yard related costs can be modeled by a vessel specific penalty function increasing from the favorite berth. Such yard cost function simplifies the reality because transshipment flows between incoming vessels are not captured. In fact, the simultaneous assignment of vessels to berths would induce a quadratic yard-related cost function. However, since the BAP planning horizon is shorter than the average container dwell time inside the yard, the BAP can assume that the majority of the outbound containers are already in the yard, and disregard this quadratic effect. Instead, in the TBAP the yard costs cannot be simplified by vessel specific cost functions, and the quadratic term must be considered.

Vessel arrival times have different meanings in the operational and in the tactical problems. In the TBAP shipping lines indicate time ranges for the expected arrival times, e.g. Monday morning with a weekly frequency. The tactical berth plan must accommodate for such arrival times or an alternative agreement should be searched. In the BAP we assume to know exactly vessel arrival times and a berth plan is drawn such that the waiting times to moor are minimized. Service quality objectives are usually achieved by imposing time windows for the berthing times that begins at the expected arrival times. Synthetically, while the BAP focuses on minimization of berth waiting times, in the TBAP we want to know if accommodating a customer request is feasible and how it impacts on the whole terminal performances such as yard costs and quay crane utilization.

The temporal aspect of the berth allocation problems depends not only on arrival times but also on the expected handling times. Handling times are influenced by many factors such as the amount of loading and unloading containers, the distribution of these containers inside the vessels, the number of quay cranes assigned to each work shift, etc. In fact, the vessel handling does not decrease proportionally augmenting the number of assigned quay cranes. This happens because of QC interferences due to safety distance when moving along a unique rail. Of course, the distribution of work-load inside the vessel is relevant too: a balanced distribution 
is favorable to the deployment of more QCs. A detailed forecast can be obtained by solving the Quay Crane Scheduling Problem (QCSP), see e.g. Kim and Park (2004), and Moccia et al. (2006), which requires a considerable amount of data available few days before vessel arrival. Therefore, at the operational level the forecast about the handling time becomes more accurate as the berthing time approaches. The BAP, thus, assumes deterministic handling times or, by integration with a QCSP module, chooses between different loading and unloading plans. The TBAP, instead, deals with the negotiation between the terminal and the shipping lines about reserved assignment of quay cranes along work shifts. For a given amount of requested quay crane hours it could be possible to propose different profiles. For example, assume that we have a request for a vessel that requires six QCs work shifts and the customer acknowledges to evaluate both an intensive QC profile (for example three QCs on two work shifts) and a longer one (two QCs on three work shifts). The terminal managers want to know the trade-off between the two profiles. The faster one will be likely more satisfying for the customer because of the smaller handling time; on the contrary, the slower one will put less pressure on the quay cranes availability, which could be a bottleneck at some periods. However, the relations are by far more complicated because if the quay cranes are not a limiting factor on the vessel expected processing time, then a faster handling time is advantageous for the terminal too because it augments berth availability. Similarly, customers can be extremely sensitive to faster handling times regarding mother vessels and less demanding for feeders. It appears clear that the so called Quay Crane Assignment Problem (QCAP), i.e. deciding how many QCs to assign and for how long, has a relevant impact on the berth allocation. In this work, we aim to combine berth allocation with QCAP and solve this new integrated problem at the tactical level from the point of view of a transshipment terminal. The purpose is to support the terminal in its negotiation process with analytic tools and quantitative results.

The remainder of this paper is organised as follows. A literature review is provided in Section 2 while the problem description as well as two formulations for the TBAP with QCs assignment are presented in Section 
3. Models' validation and results obtained with a commercial solver are illustrated in Section 4. The heuristics we propose to solve the TBAP is described in Section 5 and computational results are discussed in Section 6. Finally, we draw our conclusions in Section 7.

\section{Literature review}

The operational berth allocation problem has received so far a larger attention than the tactical one in the scientific literature. Therefore, our literature review is mainly referred to the BAP. However, we point at the shared issues between operational and tactical levels and we discuss in more detail the articles relevant to the TBAP.

The BAP consists in allocating ships to berths along a time axis. Usual side constraints are berth's allowable draft (depth of the water), time windows and priorities assigned to the ships, favorite berthing areas, etc. The BAP can be modeled as a discrete problem if the quay is viewed as a finite set of berths. In this case the berths can be described as fixed length segments, or, if the spatial dimension is ignored, as points. Continuous models consider that ships can berth anywhere along the quay. While continuous models are more realistic, discrete ones can be very useful to study relaxed problems in order to devise efficient algorithms for them.

Imai et al. (2001) have proposed the Dynamic Berth Allocation Problem (DBAP) formulation in which the quay is represented as a finite set of berthing points. This formulation is called "dynamic" as opposed to a previous one called the Static Berth Allocation Problem (SBAP), see (Imai et al., 1997), which considers the case where all ships are already in the port when the berths become available. The SBAP is solvable in polynomial time with the Hungarian method since it is reducible to an assignment problem. In their paper, the authors take advantage of this characteristic. They propose a suitable Lagrangian relaxation for the DBAP where the subproblem is an assignment problem. Their computational results show that the DBAP is easy to solve as long as the instances are "close" to the static case, in the sense that most ships are already in the port when the berths become available. The objective function is the sum of the ship 
service times. As the authors point out, this objective function does not consider ship priorities.

Nishimura et al. (2001) have presented a non-linear integer program and a genetic algorithm based on a different representation of the spatial dimension in which the quay is a collection of segments and up to two ships can share the same segment at the same time if their lengths are compatible with the length of the berth segment. Additional constraints relative to the water depth of the berths are also introduced.

The DBAP formulation was extended in Imai et al. (2003) to consider service priorities which are handled by introducing in the objective function a term corresponding to service time. Priorities, based for example on volumes, can also be incorporated in the model. The resulting formulation is non-linear. The authors show that with a suitable Lagrangian relaxation, the subproblem becomes a quadratic assignment problem. Since this problem is not well solved by exact methods, the authors have developed a genetic heuristics.

In Lim (1998) the quay is represented as a continuous line. A heuristics solves the problem of deciding the berthing points given the berthing time of the ships, assuming constant handling times. This approach does not solve the general problem in which the berthing time is a decision variable and the handling time varies along the quay.

Imai et al. (2005) address the continuous BAP with the purpose of minimizing the total service time of ships, when handling time of a ship depends on the quay location assigned to it. They present a heuristic algorithm which solves the problem in two stages, by improving the solution for the discrete case. Tests are performed on generated instances with quaylength up to $1600 \mathrm{~m}$ and up to 60 ships to be allocated.

Cordeau et al. (2005) consider both the discrete and the continuous BAP. Two formulations and two tabu search heuristics are presented and tested on realistic generated instances derived by a statistical analysis of traffic and berth allocation data of the port of Gioia Tauro (Italy).

Moorthy and Teo (2006) address the design of a berth template, a tactical planning problem that arises in transshipment hubs and concerns the allocation of favorite berthing locations (home berths) to vessels which pe- 
riodically call at the terminal. The problem is modeled as a bicriteria optimization problem, which reflects the trade-off between service levels and costs. The authors propose two procedures able to build good and robust templates, which are evaluated by simulating their performances; robust templates are also compared with optimal templates on real-life generated instances. The paper approach builds on a heuristic algorithm for the BAP presented in Dai et al. (2007).

Cordeau et al. (2007) can be regarded as introductory to the TBAP. The paper deals with the Service Allocation Problem (SAP), a tactical problem arising in the yard management of a container transhipment terminal. A service, also called port route, is the sequence of ports visited by a vessel. Shipping companies plan their port routes in order to match the demand for freight transportation. A shipping company will usually ask the terminal management to dedicate specific areas of the yard and the quay (home berths) to their services. The SAP objective is the minimization of container rehandling operations inside the yard choosing the home berth for each service. The SAP is formulated as a Generalized Quadratic Assignment Problem (GQAP, see e.g. Cordeau et al. (2006), and Hahn et al. (2008)) with side constraints, and solved by an evolutionary heuristics. The SAP can be seen as a relaxed TBAP when collapsing the temporal dimension, and disregarding the choice of QC profiles. The SAP output consists in reference home berths that planners consider when drawing the berth template.

Imai et al. (2007) consider the case of indented berths, where multiple small ships can be served by the same berth simultaneously. The problem is formulated as an integer linear problem and solved by genetic algorithms. Solutions are evaluated by comparing the indented terminal with a conventional terminal of the same size: tests on generated instances show that the total service time for all ships is longer in indented terminals, although mega-ships are served faster.

Wang and Lim (2007) propose a stochastic beam search scheme for the BAP. The implemented algorithm is tested on real-life data from the Singapore Port Terminal (the size of instances is up to 400 vessels); it outperforms state-of-the-art metaheuristics, providing better solutions in 
shorter running times.

Monaco and Sammarra (2007) propose a strong formulation for the discrete BAP as a dynamic scheduling problem on unrelated parallel machines and develop an efficient Lagrangian heuristic algorithm. Instances up to 30 ships and 7 berthing points are solved reaching near-optimal solutions in short computational time.

The integration of berth allocation and quay cranes assignment has received less attention in the scientific literature; however, a few studies on this specific topic have been recently published.

Park and Kim (2003) have firstly integrated the BAP in the continuous case with the QCAP, also considering the scheduling of quay cranes. The integrated problem is formulated as an integer program and a two-phase solution procedure is presented to solve the model. In the first phase, the berthing time and position of vessels and the number of quay-cranes assigned to each vessel at each time step are determined using Lagrangian relaxation and a subgradient optimization technique; the objective is to minimize the sum of penalty costs over all ships. In the second phase, cranes are scheduled along the quay via dynamic programming, with the objective of minimizing the number of setups. Up to 40 vessels are scheduled over a time horizon of one week, with a berth of $1200 \mathrm{~m}$ and 11 QCs available. With respect to the problem formulation, authors take into account some practical aspects such as favourite berthing positions of vessels, maximum and minimum number of cranes to be assigned to each vessel, penalty costs due to earlier or later berthing time, and later departure time (with respect to previously committed time).

Meisel and Bierwirth (2006) investigate the simultaneous allocation of berths and quay cranes, focusing on the reduction of QCs idle times, which significantly impact on terminal's labor costs. A heuristic scheduling algorithm based on priority-rules methods for the resource-constrained project scheduling is proposed and tested on six instances, based on real data, which consider up to 18 vessels to be served in two days. Preliminary results, compared to the manually generated schedules which have been used in practice, are encouraging. In this approach, each vessel represents an activity which can be performed in 8 different modes, each mode representing 
a given QC-to-Vessel assignment over time. The concept of "mode" seems analogous to the concept of profile we have introduced so far; however, no detailed description of these modes is available in the paper.

Imai et al. (2008) address the simultaneous berth-crane allocation and scheduling problem, taking into account physical constraints of quay cranes, which cannot move freely among berths as they are all mounted on the same track and cannot bypass each other. A MIP formulation which minimizes the total service time is proposed and a genetic algorithm-based heuristics is developed to find an approximate solution. Computational experiments have been performed on generated instances, which consider between 34 and 88 ships calling over a period of one week, with 4-5 berths and between 8 and 18 QCs available. As authors recognize, the relationship between the number of cranes and the handling time is not investigated in the paper; indeed, a reference number of cranes needed by each ship is assumed to be given as input of the problem.

Meisel and Bierwirth (2009) study the integration of BAP and QCAP with a focus on quay cranes productivity. An integer linear model is presented and a construction heuristics, local refinement procedures and two meta-heuristics are developed to solve the problem. Authors compare their approach to the one proposed by Park and Kim (2003) over the same set of instances and they always provide better solutions. More complex instances are generated, taking into account a time horizon of one week, a berth length of $1000 \mathrm{~m}$ and 10 QCs available to serve up to 40 vessels. Vessels are divided in 3 classes (Feeder, Medium and Jumbo) with different technical specifications and cost rates. Only small instances (20 vessels) are near-optimally solved by a commercial solver, whereas the proposed heuristics perform relatively well also on bigger instances. An analysis of quay crane's productivity losses, mainly due to interference among QCs and to the distance of the vessel berthing position from the yard areas assigned to this vessel, is also presented and their impact on the terminal's service cost is evaluated. 


\section{Mathematical Models}

In this section we firstly provide a compact description of the problem and motivate our modeling choices; in particular, in Section 3.1, we illustrate the concept of QC assignment profiles. In Section 3.2 we provide additional details regarding yard costs related to transshipment flows among ships. The described cost figures and operational parameters were provided by the Medcenter Container Terminal (MCT), port of Gioia Tauro, Italy. We then present a mixed integer quadratic programming formulation (MIQP) for the TBAP with integrated QCs assignment in Section 3.3, as well as a linearization of the MIQP model which reduces to a mixed integer linear program (MILP) in Section 3.4.

With respect to the BAP, we consider the discrete case. As announced in Section 1, the fundamental modeling tool of our formulation is the socalled quay crane profile, intended as the number of quay cranes assigned to the ship at each time step. Given $n$ ships and $m$ berths, we aim to assign, over a certain time horizon, a home berth and a QC profile to each ship as well as schedule incoming ships according to time windows on their arrival time and on berths' availabilities, in order to, on the one hand, maximize the total value of chosen QC assignment profiles and, on the other hand, minimize the housekeeping costs generated by transshipment flows between ships.

The integrated problem presents increased complexity because the ship handling time is not constant but depends on the number of quay cranes assigned to the ship. With respect to the classical BAP, this implies additional decision variables and constraints.

\subsection{QC assignment profiles}

The use of QC profiles to handle the assignment of quay cranes to ships is firstly motivated by the practice: at the tactical level and, in particular, in the context of a negotiation process between the terminal and the shipping companies, terminal's managers need to be aware of the trade-off among the different QC profiles they may propose to the counterpart.

Concerning the mathematical model, the concept of QC profiles presents 
several advantages with respect to the ability to capture real-world issues and with respect to the control that the terminal can have on several aspects during the optimization process. These are the main reasons why we have explicitly introduced this feature in the formulation.

We assume to have a set of feasible $Q C$ profiles $P_{i}$ for every ship $i \in N$, which are defined by the terminal according to the specific amount of $Q C$ hours requested by the ship (and usually legally bound by contracts) as well as internal rules and good practices related to the efficiency of operations in the terminal.

Our approach differs from the traditional modeling choice present in the literature, e.g. Park and Kim (2003), Imai et al. (2008), Meisel and Bierwirth (2009), which usually assigns quay cranes hour by hour, without any control on the final outcome in terms of QC profiles, according to their models. As mentioned, the concept of "mode" in Meisel and Bierwirth (2006) is somehow similar to our concept of QC profile, but the authors do not provide enough details to allow comparisons.

For a given vessel, feasible QC profiles usually vary in length (number of shifts) as well as in the distribution of $Q C$ cranes over the active shifts, in order to ensure the requested amount of QC hours.

Some operational constraints, which are usually not taken into account by other models, can be directly integrated in the definition of the set of feasible profiles. A common rule, for instance, is that quay cranes are assigned to vessels and placed on the corresponding quay segment shift by shift: this means that a quay crane cannot be moved from one vessel to another at whatever moment, but only between two shifts. This constraint can be easily handled by forcing profiles to maintain exactly the same number of quay cranes during a shift. Another good practice is to keep the distribution of quay cranes as much regular as possible among active shifts; a variance of one or at most two QCs can be considered acceptable, although high variability should be avoided as much as possible. Also this feature can be included in our profile set definition easily.

In addition to these general rules, the terminal can manage more directly some priority-related issues. Since the set of feasible QC profiles is defined for every ship, managers can assign different minimum and maxi- 
mum handling times not only depending on the ship's size and the traffic volume but also depending on the ship's relative importance for the terminal. This also applies for the minimum and maximum number of quay cranes allowed to be assigned to a given ship. We would like to remark that this is an important advantage provided by our approach, compared to other models in the literature where handling time is either considered an input of the problem or barely controlled by time windows on the vessel's arrival and departure, in addition to some priority-related weights in the objective function, which usually aim to serve faster vessels with high priority. Furthermore, each QC profile has an associated "value" which reflects technical aspects such as the resources utilized by the profile itself but which is also computed by taking into account the specific vessel which will use the profile; in other words, the same QC profile i.e. same length and QCs distribution over time, can have different values associated to different ships, according indeed to their priority or importance.

With respect to productivity losses due to quay cranes interference, recently studied by Meisel and Bierwirth (2009), we can easily include this feature in the definition of the feasible set of profiles. Indeed, we can use the approach suggested by the authors to compute, for each profile, the actual quay crane productivity instead of the theoretical one.

It is also worth a remark concerning the time: in our model the time horizon, and thus every working shift, is discretized in time steps and we allow a profile to start at every time step of the shift. However, since we assume that a vessel starts to be operated when it arrives at the port, the starting time of the profile assigned to the vessel by any feasible solution must comply with the arrival time of the vessel itself at the port (which is also a decision variable of the problem).

In order to improve understanding of the QC profile concept, and its relation with the integration between Berth and QC Allocation planning, we provide in Fig. 1 an example of such plan referred to the scheduling and assignment of 5 vessels to 3 berths over a time horizon of 8 working shifts. The example can be read very easily. Consider, for instance, the Ship 1: it berths at shift 1, and three QCs are allocated to it for carrying out operations during the same shift; next, at working shifts 2 and 3, Ship 1 


\begin{tabular}{|c|c|c|c|c|c|c|c|c|}
\hline TIME & $w s=1$ & $w s=2$ & $w s=3$ & $w s=4$ & $w s=5$ & $w s=6$ & ws $=7$ & $w S=8$ \\
\hline \multirow{2}{*}{ berth 1} & \multicolumn{3}{|c|}{ ship 1} & & \multicolumn{4}{|c|}{ ship 2} \\
\hline & 3 & 2 & 2 & & 4 & 4 & 5 & 5 \\
\hline \multirow{2}{*}{ berth 2} & & \multicolumn{2}{|c|}{ ship 3} & & & \multicolumn{3}{|c|}{ ship 4} \\
\hline & & 4 & 5 & & & 3 & 3 & 3 \\
\hline \multirow{2}{*}{ berth 3} & & & \multicolumn{5}{|c|}{ ship 5} & \\
\hline & & & 3 & 3 & 3 & 2 & 2 & \\
\hline QCs & 3 & 6 & 10 & 3 & 7 & 9 & 10 & 8 \\
\hline
\end{tabular}

Figure 1: Example of a Berth \& Quay Cranes Allocation Plan.

remains berthed, but one QC is de-allocated, with only two QCs remaining allocated to the ship. At the end of shift 3 operations terminate and the ship is released. Of course, analogous comments hold for the other 4 ships.

Finally, as for the value of each QC profile, it should be noted that the terminal faces different customer sensitivities to the $\mathrm{QC}$ intensity of contracted profiles. In fact, given two reference profiles, one for mother vessels and one for feeders, the added value of shortening the same handling time by selecting a more QC intensive profile is higher (double as order of magnitude) for a mother vessel than for a feeder. Furthermore, the feasible profiles span different ranges for the two classes of vessels: with respect to the same reference handling volume, we can have acceptable profiles which are slower for the feeders than for a mother vessel. Fig. 22 illustrates these patterns assuming that the reference profile extends on five work shifts (30 hours) for a mother vessel and on three work shifts (18 hours) for a feeder. We remark that for privacy reasons, the actual monetary figures cannot be presented, hence the picture has only a qualitative meaning. 


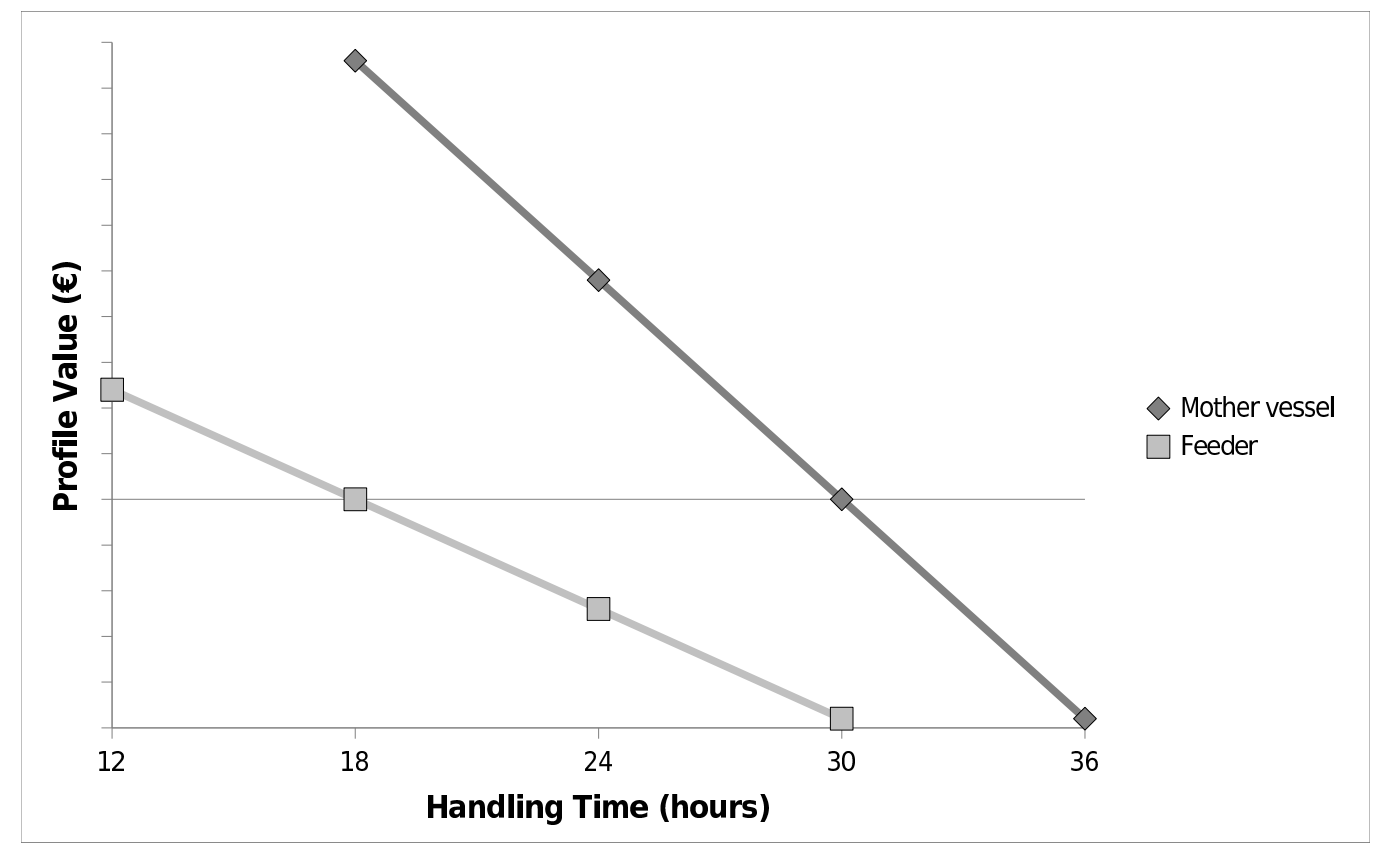

Figure 2: QC profile range and value variation according to the handling time and class of vessel.

\subsection{Transshipment-related yard costs}

When loading (or unloading) a vessel the containers must be at (or allocated to) yard positions close enough to the vessel berthing point in order to speed up the vessel handling. Usually, at the Medcenter Container Terminal (MCT) of the port of Gioia Tauro, a yard position is evaluated as satisfyingly close to a berth if the distance along the quay axis is less than 600 meters. We remark that this maximal close distance value can be lowered for higher priority workloads. Furthermore, when we estimate yard-related transshipment costs induced by berth allocation, we do not consider the real yard position of the loading and unloading containers. In fact, we assume that the expected travelled distance along the quay axis is given by the distance between the incoming and outgoing berths. If this distance is lower than the threshold value of 600 meters, then a container will likely move from the quay to its assigned yard position when unload- 
ing and from this yard position to the quay when loading. However, in a large transshipment terminal, such as the one at the Gioia Tauro port, the distance between the unloading berth and the loading one is often larger than 600 meters. Therefore, containers are moved before the arrival of the outgoing vessel from their current yard positions to new ones closer to the outgoing berth. This process is called housekeeping and requires a dedicated management in order to accommodate operational constraints like the capacity of the yard positions, the maximum container handling workload for a given work shift, etc. A rule motivated by cost minimization enforces that whenever the distance along the quay axis is larger than 1100 meters, the yard-to-yard transfer is operated by deploying multi trailer vehicles instead of straddle carries. Therefore we have a yard cost function that depends upon the distance between the incoming and outgoing berths according to three transport modalities:

- the distance is below 600 meters: no housekeeping is performed, the unitary transport cost, euro/(meter x container), depends upon straddle carriers cost figures only;

- the distance is between 600 and 1100 meters: a housekeeping process is activated by deploying straddle carriers only, however we face a transport cost larger than in the previous distance range;

- the distance is larger than 1100 meters: the housekeeping is performed by using the less expensive multi trailer vehicles (higher capacity than the straddle carriers).

The qualitative pattern of this piecewise linear cost function is given in Fig. 3, where we indicate by SC the direct transfer with straddle carriers, by $\mathrm{HK}$ SC the housekeeping with straddle carriers, and by $\mathrm{HK} \mathrm{MT}$ the housekeeping with multi trailer vehicles.

\subsection{MIQP Formulation}

In this section we present a mixed integer quadratic programming formulation for the TBAP with QCs assignment. Input data for this problem are: 


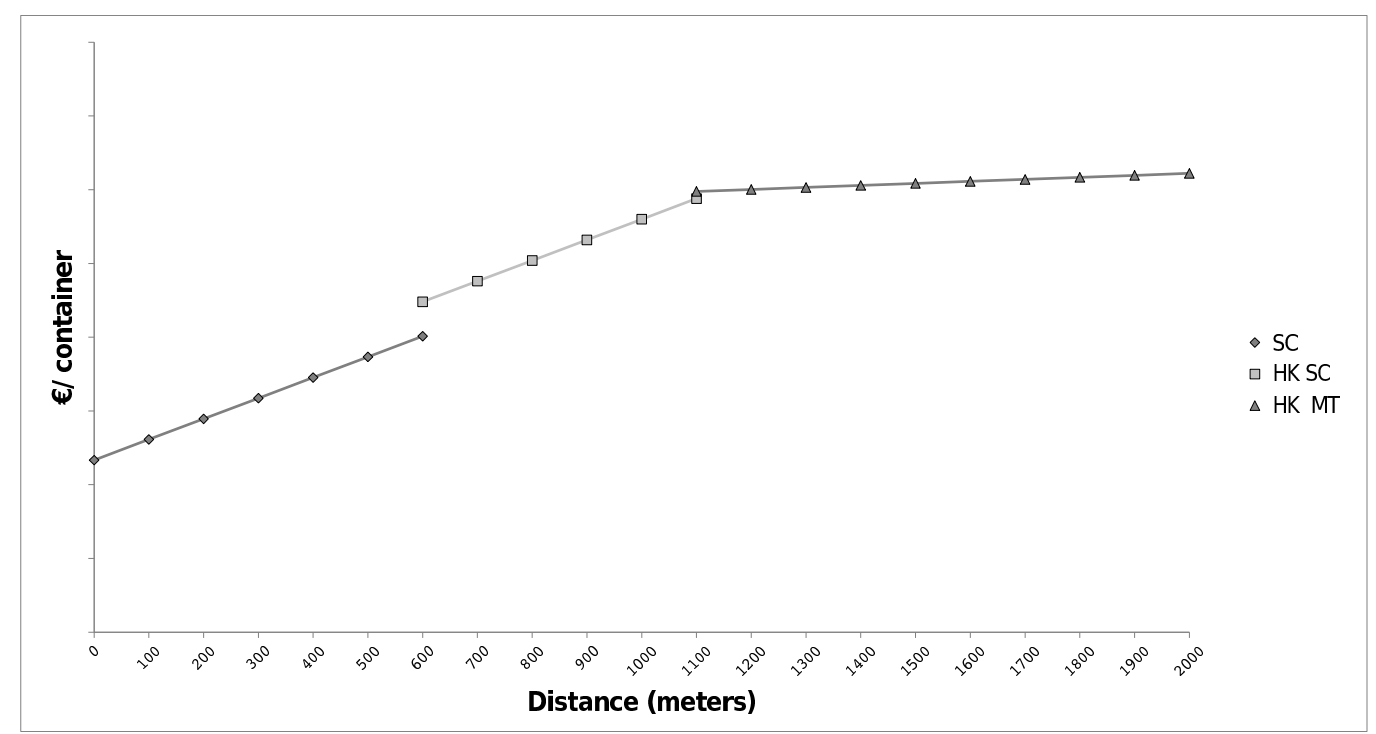

Figure 3: Yard costs according to the distance between the incoming and outgoing berths.

$N \quad$ set of vessels, with $|N|=n$;

$M \quad$ set of berths, with $|M|=m$;

$H$ set of time steps (each time step $h \in H$ is submultiple of the work shift length);

$S \quad$ set of the time step indexes $\{1, \ldots, \bar{s}\}$ relative to a work shift; $\bar{s}$ represents the number of time steps in a work shift;

$\mathrm{H}^{\mathrm{s}} \quad$ subset of $\mathrm{H}$ which contains all the time steps corresponding to the same time step $s \in S$ within a work shift;

$P_{i}^{s} \quad$ set of feasible quay crane assignment profiles for the vessel $i \in N$ when vessel arrives at a time step with index $s \in S$ within a work shift;

$P_{i} \quad$ set of quay crane assignment profiles for the vessel $i \in N$, where $P_{i}=\cup_{s \in S} P_{i}^{s}$;

$t_{i}^{p} \quad$ handling time of $\operatorname{ship} i \in N$ under the $Q C$ profile $p \in P_{i}$ expressed as multiple of the time step length;

$\nu_{i}^{p} \quad$ the value of serving the ship $i \in N$ by the quay crane profile $p \in P_{i}$;

$q_{i}^{p u}$ number of quay cranes assigned to the vessel $i \in N$ under the profile $p \in P_{i}$ at the time step $u \in\left(1, \ldots, t_{i}^{p}\right)$, where $u=1$ corresponds to the ship arrival time;

$\mathrm{Q}^{\mathrm{h}} \quad$ maximum number of quay cranes available at the time step $h \in H$;

$f_{i j} \quad$ number of containers exchanged between vessels $i, j \in N$; 
$d_{k w} \quad$ unit housekeeping cost between yard slots corresponding to berths $k, w \in M$;

$\left[a_{i}, b_{i}\right] \quad$ [earliest, latest] feasible arrival time of ship $i \in N$;

$\left[a^{k}, b^{k}\right] \quad$ [start, end] of availability time of berth $k \in M$;

$\left[\mathrm{a}^{\mathrm{h}}, \mathrm{b}^{\mathrm{h}}\right] \quad$ [start, end] of the time step $\mathrm{h} \in \mathrm{H}$.

We define a graph $\mathrm{G}^{\mathrm{k}}=\left(\mathrm{V}^{\mathrm{k}}, \mathrm{A}^{\mathrm{k}}\right) \forall \mathrm{k} \in \mathrm{M}$, where $\mathrm{V}^{\mathrm{k}}=\mathrm{N} \cup\{\mathrm{o}(\mathrm{k}), \mathrm{d}(\mathrm{k})\}$, with $\mathrm{o}(\mathrm{k})$ and $\mathrm{d}(\mathrm{k})$ additional vertices representing berth $k$, and $A^{k} \subseteq$ $\mathrm{V}^{\mathrm{k}} \times \mathrm{V}^{\mathrm{k}}$. The following decision variables are defined:

- $x_{i j}^{k} \in\{0,1\} \forall k \in M, \forall(i, j) \in A^{k}$, set to 1 if ship $j$ is scheduled after ship $i$ at berth $k$, and 0 otherwise;

- $y_{i}^{k} \in\{0,1\} \forall k \in M, \forall i \in N$, set to 1 if ship $i$ is assigned to berth $k$, and 0 otherwise;

- $\gamma_{i}^{h} \in\{0,1\} \forall h \in H, \forall i \in N$, set to 1 if ship $i$ arrives at time step $h$, and 0 otherwise;

- $\lambda_{i}^{p} \in\{0,1\} \forall p \in P_{i}, \forall i \in N$, set to 1 if ship $i$ is served by the profile $p$, and 0 otherwise;

- $\rho_{i}^{\text {ph }} \in\{0,1\} \forall p \in P_{i}, \forall h \in H, \forall i \in N$, set to 1 if ship $i$ is served by profile $p$ and arrives at time step $h$, and 0 otherwise;

- $\mathrm{T}_{i}^{k} \geq 0 \forall \mathrm{k} \in M, \forall i \in \mathrm{N}$, representing the berthing time of ship $i$ at the berth $k$, i.e. the time when the ship moors;

- $\mathrm{T}_{\mathrm{o}(\mathrm{k})}^{\mathrm{k}} \geq 0 \forall \mathrm{k} \in \mathrm{M}$, representing the starting operation time of berth $k$, i.e. the time when the first ship moors at the berth;

- $\mathrm{T}_{\mathrm{d}(\mathrm{k})}^{\mathrm{k}} \geq 0 \quad \forall \mathrm{k} \in \mathrm{M}$, representing the ending operation time of berth $\mathrm{k}$, i.e. the time when the last ship departs from the berth. 
The TBAP with QC assignment can therefore be formulated as follows:

$$
\begin{aligned}
& \max \sum_{i \in N} \sum_{p \in P_{i}} v_{i}^{p} \lambda_{i}^{p}-\frac{1}{2} \sum_{i \in N} \sum_{k \in M} y_{i}^{k} \sum_{j \in N} \sum_{w \in M} f_{i j} d_{k w} y_{j}^{w} \\
& \text { s.t. } \sum_{k \in M} y_{i}^{k}=1 \quad \forall i \in N \text {, } \\
& \sum_{j \in N \cup\{d(k)\}} x_{o(k), j}^{k}=1 \quad \forall k \in M \\
& \sum_{i \in N \cup\{o(k)\}} x_{i, d(k)}^{k}=1 \quad \forall k \in M, \\
& \sum_{j \in \mathrm{N} \cup\{\mathrm{d}(\mathrm{k})\}} x_{i j}^{k}-\sum_{j \in \mathrm{N} \cup\{\mathbf{o}(\mathrm{k})\}} x_{j i}^{k}=0 \quad \forall k \in M, \forall i \in N, \\
& \sum_{j \in N \cup\{d(k)\}} x_{i j}^{k}=y_{i}^{k} \quad \forall k \in M, \forall i \in N, \\
& \mathrm{~T}_{i}^{\mathrm{k}}+\sum_{\mathrm{p} \in \mathrm{P}_{i}} \mathrm{t}_{i}^{\mathrm{p}} \lambda_{i}^{p}-\mathrm{T}_{j}^{\mathrm{k}} \leq\left(1-x_{i j}^{\mathrm{k}}\right) M \quad \forall \mathrm{k} \in M, \forall i \in \mathrm{N}, \forall j \in \mathrm{N} \cup\left\{\mathrm { d } \left(\mathrm{k}\left(\mathrm{p}_{j}\right)\right.\right. \\
& \mathrm{T}_{\mathrm{o}(\mathrm{k})}^{\mathrm{k}}-\mathrm{T}_{\mathrm{j}}^{\mathrm{k}} \leq\left(1-x_{\mathrm{o}(\mathrm{k}), \mathrm{j}}^{\mathrm{k}}\right) \mathrm{M} \quad \forall \mathrm{k} \in \mathrm{M}, \forall \mathrm{j} \in \mathrm{N}, \\
& a_{i} y_{i}^{k} \leq T_{i}^{k} \quad \forall k \in M, \forall i \in N \text {, } \\
& \mathrm{T}_{i}^{\mathrm{k}} \leq \mathrm{b}_{i} \mathrm{y}_{\mathrm{i}}^{\mathrm{k}} \quad \forall \mathrm{k} \in \mathrm{M}, \forall i \in \mathrm{N} \text {, } \\
& \mathrm{a}^{\mathrm{k}} \leq \mathrm{T}_{\mathrm{o}(\mathrm{k})}^{\mathrm{k}} \quad \forall \mathrm{k} \in \mathrm{M} \text {, } \\
& \mathrm{T}_{\mathrm{d}(\mathrm{k})}^{\mathrm{k}} \leq \mathrm{b}^{\mathrm{k}} \quad \forall \mathrm{k} \in \mathrm{M} \text {, } \\
& \sum_{p \in P_{i}} \lambda_{i}^{p}=1 \quad \forall i \in N \text {, } \\
& \sum_{h \in H^{s}} \gamma_{i}^{h}=\sum_{p \in P_{i}^{s}} \lambda_{i}^{p} \quad \forall i \in N, \forall s \in S, \\
& \sum_{k \in M} T_{i}^{k}-b^{h} \leq\left(1-\gamma_{i}^{h}\right) M \quad \forall h \in H, \forall i \in N, \\
& \mathrm{a}^{\mathrm{h}}-\sum_{\mathrm{k} \in \mathrm{M}} \mathrm{T}_{\mathrm{i}}^{\mathrm{k}} \leq\left(1-\gamma_{i}^{\mathrm{h}}\right) \mathrm{M} \quad \forall \mathrm{h} \in \mathrm{H}, \forall \mathrm{i} \in \mathrm{N}, \\
& \rho_{i}^{p h} \geq \lambda_{i}^{p}+\gamma_{i}^{h}-1 \quad \forall h \in H, \forall i \in N, \forall p \in P_{i}, \\
& \sum_{i \in N} \sum_{p \in P_{i}} \sum_{u=\max \left\{h-t_{i}^{p}+1 ; 1\right\}}^{h} \rho_{i}^{p u} \mathrm{q}_{i}^{p(h-u+1)} \leq Q^{h} \quad \forall h \in H^{\bar{s}} \text {, }
\end{aligned}
$$




$$
\begin{aligned}
x_{i j}^{k} \in\{0,1\} & \forall k \in M, \forall(i, j) \in A^{k}, \\
y_{i}^{k} \in\{0,1\} & \forall k \in M, \forall i \in N, \\
\gamma_{i}^{h} \in\{0,1\} & \forall h \in H, \forall i \in N, \\
\lambda_{i}^{p} \in\{0,1\} & \forall p \in P_{i}, \forall i \in N, \\
\rho_{i}^{\text {ph }} \in\{0,1\} & \forall p \in P_{i}, \forall h \in H, \forall i \in N, \\
T_{i}^{k} \geq 0 & \forall k \in M, \forall i \in N \cup\{o(k), d(k)\} .
\end{aligned}
$$

The objective function (11) maximizes the sum of the values of the chosen quay crane assignment profiles over all the vessels and simultaneously minimizes the yard-related housekeeping costs generated by the flows of containers exchanged between vessels. Constraints (21) state that every ship $i$ must be assigned to one and only one berth $k$. Constraints (3) and (4) define the outgoing and incoming flows to the berths, while flow conservation for the remaining vertices is ensured by constraints (5). Constraints (6) state the link between variables $x_{i j}^{k}$ and $y_{i}^{k}$, while precedences in every sequence are ensured by constraints (77) and (8), which coherently set time variables $\mathrm{T}_{i}^{\mathrm{k}}$. Time windows on the arrival time are stated for every ship by constraints (9) and (10), while time windows on berths' availabilities are stated by constraints (11) and (12). Constraints (13) ensure that one and only one QCs profile is assigned to every ship. Constraints (14) define the link between variables $\gamma_{i}^{h}$ and $\lambda_{i}^{p}$ while constraints (15) and (16) link binary variables $\gamma_{i}^{h}$ to the arrival time $T_{i}^{k}$. Observe that constraints (10) imply $T_{i}^{k}=0$ when ship $i \in N$ does not moor at berth $k \in K$. Variables $\rho_{i}^{\text {ph }}$ are linked to variables $\lambda_{i}^{p}$ and $\gamma_{i}^{h}$ by constraints (17): in particular, $\rho_{i}^{\text {ph }}$ is equal to 1 if and only if $\lambda_{i}^{p}=\gamma_{i}^{h}=1$. Finally, constraints (18) ensure that, at every time step, the total number of assigned quay cranes does not exceed the number of quay cranes which are available in the terminal.

To better illustrate capacity constraints (18), we come back to the example shown in Fig. 1, which refers to the scheduling and assignment of $|\mathrm{N}|=5$ vessels to $|\mathrm{M}|=3$ berths over a time horizon of $|\mathrm{H}|=8$ time steps. Here we assume that a time step corresponds to one working shift. From the plan we can infer the following non-zero data: 


$$
\begin{aligned}
& i=1 \quad \rho_{1}^{p 1}=1 \quad t_{1}^{p}=3 \quad q_{1}^{p 1}=3, q_{1}^{p 2}=2, q_{1}^{p 3}=2 \\
& i=2 \quad \rho_{2}^{\mathrm{p} 5}=1 \quad \mathrm{t}_{2}^{\mathrm{p}}=4 \quad \mathrm{q}_{2}^{\mathrm{p} 1}=4, \mathrm{q}_{2}^{\mathrm{p} 2}=4, \mathrm{q}_{2}^{\mathrm{p} 3}=5, \mathrm{q}_{2}^{\mathrm{p} 4}=5 \\
& i=3 \quad \rho_{3}^{\mathrm{p} 2}=1 \quad \mathrm{t}_{3}^{\mathrm{p}}=2 \quad \mathrm{q}_{3}^{\mathrm{p} 1}=4, \mathrm{q}_{3}^{\mathrm{p} 2}=5 \\
& i=4 \quad \rho_{4}^{p 6}=1 \quad t_{4}^{p}=3 \quad q_{4}^{p 1}=3, \quad q_{4}^{p 2}=3, q_{4}^{p 3}=3 \\
& i=5 \quad \rho_{5}^{p 3}=1 \quad t_{5}^{p}=5 \quad q_{5}^{p 1}=3, q_{5}^{p 2}=3, q_{5}^{p 3}=3, q_{5}^{p 4}=2, q_{5}^{p 5}=2
\end{aligned}
$$

For each time step $h=1, \ldots 8$, the corresponding constraint in (18) counts the number of active quay cranes. Let us consider the case $h=3$ : the index $u$ changes its range for each vessel, because, starting from $h=3$, it goes backwards until the beginning of the profile. Therefore we have:

$$
\begin{array}{ll}
i=1 & u=1,2,3 \\
i=2 & u=1,2,3 \\
i=3 & u=2,3 \\
i=4 & u=1,2,3 \\
i=5 & u=1,2,3
\end{array}
$$

We remark that vessels $i=2,4$ do not contribute to the sum, since $\rho_{2}^{\text {pu }}=$ $\rho_{4}^{p u}=0 \forall u=1,2,3$ and this is coherent with the plan. For the remaining vessels, $\rho_{i}^{\text {pu }}$ is not zero only for one value $u^{*}$ :

$$
\begin{array}{lll}
i=1 & u^{*}=1 & \Longrightarrow q_{1}^{p(3-1+1)}=q_{1}^{p 3}=2 \\
i=3 & u^{*}=2 \quad \Longrightarrow q_{3}^{p(3-2+1)}=q_{3}^{p 2}=5 \\
i=5 & u^{*}=3 \quad \Longrightarrow q_{5}^{p(3-3+1)}=q_{5}^{p 1}=3
\end{array}
$$

Therefore the sum in (18) reduces to:

$$
\mathrm{q}_{1}^{\mathrm{p} 3}+\mathrm{q}_{3}^{\mathrm{p} 2}+\mathrm{q}_{5}^{\mathrm{p} 1}=2+5+3=10
$$

which is indeed the total number of quay cranes which are active at time step $h=3$.

Finally, we observe that the TBAP formulation (1)-(24) can be interpreted as a Multi-Depot Vehicle Routing Problem with Time Windows (MDVRPTW), see e.g. Cordeau et al. (2005), with an additional quadratic component in the objective function and side constraints.

\subsection{MILP Formulation}

The quadratic objective function (1) can be linearized by defining an additional decision variable $z_{i j}^{k w} \in\{0,1\} \forall i, j \in N, \forall k, w \in M$, which is equal 
to 1 if $y_{i}^{k}=y_{j}^{w}=1$ and 0 otherwise. Variables $z_{i j}^{k w}$ are linked to variables $y_{i}^{k}$ by the following additional constraints:

$$
\begin{aligned}
\sum_{k \in K} \sum_{w \in K} z_{i j}^{k w}=g_{i j} & \forall i, j \in N, \\
z_{i j}^{k w} \leq y_{i}^{k} & \forall i, j \in N, \forall k, w \in M \\
z_{i j}^{k w} \leq y_{j}^{w} & \forall i, j \in N, \forall k, w \in M
\end{aligned}
$$

where $g_{i j}$ is a constant which is equal to 1 if $f_{i j}>0$ and 0 otherwise.

TBAP can therefore be formulated as a mixed integer linear program as follows:

$$
\begin{array}{cc}
\max & \sum_{i \in N} \sum_{p \in P_{i}} v_{i}^{p} \lambda_{i}^{p}-\frac{1}{2} \sum_{i \in N} \sum_{j \in N} \sum_{k \in M} \sum_{w \in M} f_{i j} d_{k w} z_{i j}^{k w} \\
\text { s.t. } & \text { (2) }-(\underline{24}),(25)-(27) .
\end{array}
$$

\section{Model validation}

In this section we describe the validation process of our models. We firstly illustrate how realistic test instances have been generated and we then present results obtained through a commercial solver.

\subsection{Generation of test instances}

Our tests are based on real data provided by MCT. We had access to historical berth allocation plans and quay cranes assignment plans concerning about 60 vessels per week over a time horizon of one month; specific information on vessels such as the arrival time and the total number of containers to be handled were also provided. Furthermore, data referring to the flows of containers exchanged between ships as well as a study on the yard-related transshipment costs were available.

Instances generated to validate our models rely on these real data. The quay, which is $3395 \mathrm{~m}$ long, is partitioned in 13 berthing points, which are equipped with 25 quay cranes (22 gantry cranes and 3 mobile cranes). The matrix of distances $\left[\mathrm{d}_{\mathrm{kw}}\right]$ is a $13 \times 13$ matrix which takes into account the 
costs estimated by the terminal to move containers between two berthing positions. Several matrices of flows $\left[f_{i j}\right]$ are generated accordingly to the distributions of containers reported on the historical data. As usual, we distinguish between feeders and mother vessels: the traffic volume is mostly influenced by the proportion between these two classes, since mother vessels present a number of loading/unloading containers in average higher than feeders. Time windows for the ships' arrival are generated accordingly to the historical data. Berths are assumed to be available for the whole time horizon, which we set to one week. A working day is divided in 4 shifts of 6 hours each, for a total of 56 time steps of 3 hours.

The sets of feasible profiles have been synthetically generated in accordance with operational rules and good practices in use at the MCT terminal. As illustrated in Table 1, we fix a set of parameters for each ship class to which a profile must comply with in order to be feasible: namely, the minimum and the maximum number of QCs to be assigned to each vessel per shift as well as the minimum and the maximum handling time (HT) allowed for each class. We consider a crane productivity of 24 containers per hours and we therefore obtain, per each class, a minimum and a maximum number of containers (column "volume" in the table): vessels' traffic volumes must comply with these ranges, according to the class they belong to. Furthermore, for all classes, a variation of at most $1 \mathrm{QC}$ is allowed between a shift and the subsequent; profiles can start either at the beginning of the shift or in the middle of the shift.

Once the whole feasible set has been generated for each class, profiles are assigned to vessels accordingly to the QC hours they need to be operated. At this point, a monetary value is associated to the couple (vessel,profile) with respect to the number of containers to be handled. This value is then adjusted by taking into account the profile's length and the utilized

\begin{tabular}{cccccc} 
Class & $\min$ QC & $\max$ QC & $\min$ HT & $\max$ HT & volume (min,max) \\
\hline Mother & 3 & 5 & 3 & 6 & $(1296,4320)$ \\
Feeder & 1 & 3 & 2 & 4 & $(288,1728)$
\end{tabular}

Table 1: Parameters for the profile set's generation. 
resources with respect to the average case.

To validate our model, we considered 6 classes of instances:

- 10 ships and 3 berths, 1 week, 8 quay cranes;

- 20 ships and 5 berths, 1 week, 13 quay cranes;

- 30 ships and 5 berths, 1 week, 13 quay cranes;

- 40 ships and 5 berths, 2 weeks, 13 quay cranes;

- 50 ships and 8 berths, 2 weeks, 13 quay cranes;

- 60 ships and 13 berths, 2 weeks, 13 quay cranes.

For each class, we generated 12 instances, with high $(\mathrm{H})$ and low $(\mathrm{L})$ traffic volumes. Each scenario is tested with a set of $\bar{p}=10,20,30$ feasible profiles for each ship. We remark that, by construction, instances of size $\bar{p}=10$ are included in instances of size $\bar{p}=20$, which are included in instances of size $\bar{p}=30$. Thus, any feasible solution for $\bar{p}=10$ is also feasible for $\bar{p}=20,30$ and so on.

\subsection{Computational results}

The MIQP and MILP formulations have been tested with CPLEX 10.2, with emphasis on the feasibility of the solution.

Time limit for instances $10 \times 3$ is 1 hour; instances $20 \times 5$ and $30 \times 5$ have a time limit of 2 hours; instances $40 \times 5,50 \times 8,60 \times 13$ have a time limit of 3 hours.

Results are illustrated in Table 2, We report only instances for which CPLEX has found a feasible solution, at least. Surprisingly, no feasible solution was found for classes $30 \times 5,50 \times 8$ and $60 \times 13$; however, an upper bound is always provided.

The objective function value is scaled to 100 with respect to the upper bound via the formula:

$$
\text { scaled obj }=\frac{o b j * 100}{U B}
$$




\begin{tabular}{lcc|lcc}
\multicolumn{3}{c}{ 10x3 } \\
\hline Instance & MILP & MIQP & Instance & MILP & MIQP \\
\hline H1_10 & 99.17 & 98.90 & L1_10 & 97.68 & 100.00 \\
H1_20 & 97.91 & 97.96 & L1_20 & 100.00 & 99.76 \\
H1_30 & 97.98 & 98.76 & L1_30 & 98.64 & 99.99 \\
H2_10 & 98.87 & 99.26 & L2_10 & 98.82 & 99.63 \\
H2_20 & 96.97 & 96.91 & L2_20 & 99.42 & 99.06 \\
H2_30 & 96.79 & - & L2_30 & 99.08 & 100.00 \\
\hline \multicolumn{5}{c}{} \\
\hline Instance & MILP & MIQP & Instance & MILP & MIQP \\
\hline H1_10 & 94.33 & - & L1_10 & 94.92 & - \\
H1_20 & 93.74 & - & L1_20 & 94.47 & - \\
H2_10 & 93.52 & 96.66 & L2_20 & 94.93 & - \\
L2_10 & 93.87 & 96.74 & L2_30 & 94.61 & - \\
\hline
\end{tabular}

Table 2: Scaled objective function of the best feasible solutions found by CPLEX in the allowed time limit.

A value of 100 means that the solution is certified to be optimal.

With respect to class 10x3, 3 out of 12 instances are solved at optimum; both MILP and MIQP formulations provide near-optimal solutions, with an average of 98.44 and 99.11 respectively.

With respect to class $20 \mathrm{x} 5$, a feasible solution is found for 4 instances out of 12 with the MILP formulation, while, using the MIQP formulation, we get a feasible solution only for 2 instances. The quality of the solution is lower, with an average of 93.87 for MILP and of 96.70 for MIQP.

Class $40 \times 5$ is only solved using the MILP formulation; a feasible solution is found for 4 instances out of 12 , with an average quality of the solution of 94.73 .

With respect to the upper bounds, we remark that the MILP formulation provides far better upper bounds than MIQP, as illustrated in Table 3.

Results clearly show that the problem is difficult to solve as-it-is already 


\begin{tabular}{lll|llll}
\multicolumn{5}{c}{ 30x5 } \\
\hline Instance & MILP UB & MIQP UB & Instance & MILP UB & MIQP UB \\
\hline H1_10 & 1754291 & 2288451 & H1_10 & 3227542 & 5939357 \\
H1_20 & 1754633 & 2288793 & H1_20 & 3228422 & 6038925 \\
H1_30 & 1754669 & 2288829 & H1_30 & 3228709 & 5941943 \\
\hline H2_10 & 1708485 & 2256299 & H2_10 & 3130833 & 5965539 \\
H2_20 & 1709020 & 2256834 & H2_20 & 3131431 & 5966137 \\
H2_30 & 1709230 & 2257044 & H2_30 & 3131677 & 5966383 \\
\hline L1_10 & 1420485 & 1787983 & L1_10 & 3014276 & 5668646 \\
L1_20 & 1420713 & 1817824 & L1_20 & 3014877 & 5669247 \\
L1_30 & 1420819 & 1842700 & L1_30 & 3015054 & 5669424 \\
\hline L2_10 & 1613252 & 1948130 & L2_10 & 3084415 & 5749854 \\
L2_20 & 1613769 & 1973914 & L2_20 & 3085121 & 5750560 \\
L2_30 & 1613805 & 2008053 & L2_30 & 3085364 & 5750803 \\
\hline
\end{tabular}

Table 3: Upper bounds provided by CPLEX using MILP and MIQP formulations.

on small instances. CPLEX or any other commercial solver are not a viable way to solve TBAP with quadratic costs, especially on bigger instances like those we expect to have in a tactical problem. We therefore developed a heuristic algorithm, which is illustrated in the next section.

\section{A bi-level heuristics for TBAP}

Our heuristic algorithm is organized in two stages: firstly, we identify a set of a QC profiles for the ships; secondly, we solve the resulting berth allocation problem for the given $\mathrm{QC}$ assignment. This procedure is repeated for several sets of $\mathrm{QC}$ profiles, which are chosen, iteration by iteration, using the traditional reduced costs arguments of mathematical programming. A scheme of the heuristic algorithm for TBAP is outlined in Fig. 4,

The initial step consists in assigning a QC profile to each ship. The maximum value profiles are chosen (ties are broken arbitrarily). This is equivalent to assign binary values to variables $\lambda$ such that equations (13) 


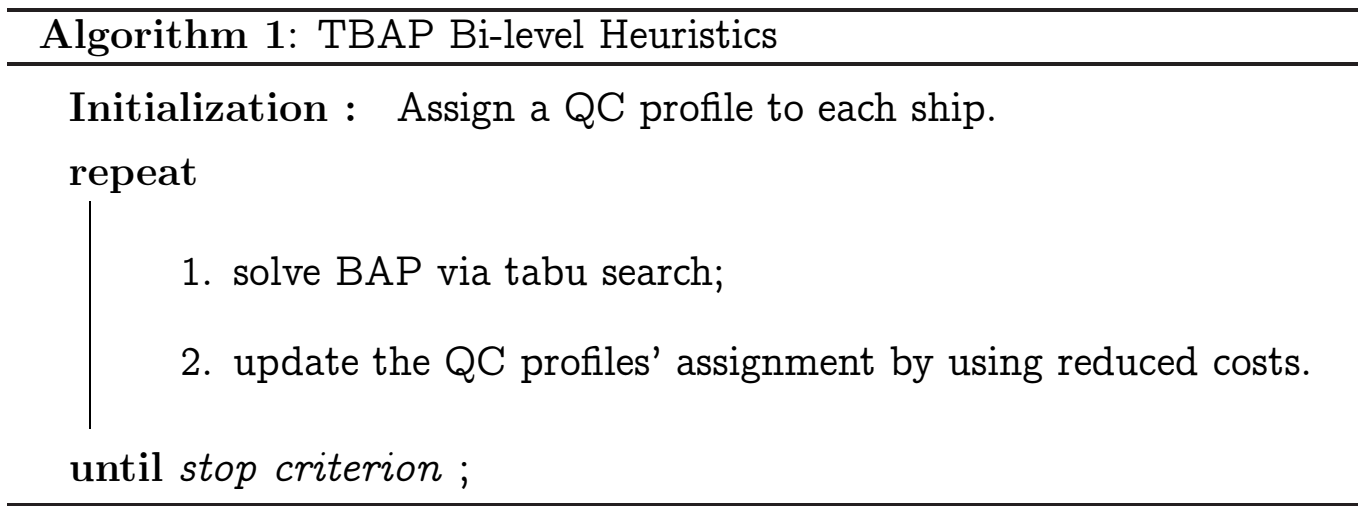

Figure 4: Scheme of the heuristic algorithm for TBAP.

are satisfied. Once the first QC assignment has been done, the bi-level procedure starts.

Given a profiles' assignment, the TBAP reduces to the berth allocation problem, with additional constraints due to the QC total capacity. We developed a tabu search algorithm which solves the BAP, aiming to minimize the yard-related transshipment housekeeping cost:

$$
\frac{1}{2} \sum_{i \in N} \sum_{k \in M} y_{i}^{k} \sum_{j \in N} \sum_{w \in M} f_{i j} d_{k w} y_{j}^{w}
$$

We remark that we take into account only the quadratic term of the TBAP objective function in (1) since, for a given QC profiles' assignment, the total value of profiles is constant. The tabu search algorithm for the BAP is illustrated in Section 5.1.

The new set of profiles is then determined using the reduced costs of variables $\lambda$, whose estimation is illustrated in Section 5.2 .

\subsection{Tabu search for BAP}

Our tabu search heuristics is an adaptation of the one of Cordeau et al. (2005) for the BAP. However, while in Cordeau et al. (2005) the function to be minimized is the weighted sum for every ship of the service time 
in the port, our heuristics minimizes the yard-related housekeeping costs generated by the flows of containers exchanged between vessels. Another difference is the handling of the side constraints concerning the QC availability: indeed, for a given assignment of QC profiles (vector $\lambda$ ), our tabu search must take into account the QC capacity constraints (18).

Denote by $S$ the set of solutions that satisfy constraints (21) - (9) and (11). The heuristics explores the solution space by moving at each iteration from the current solution $s$ to the best solution in its neighborhood $N(s)$. Each solution $s \in S$ is represented by a set of $m$ berth sequences such that every ship belongs to exactly one sequence. This solution may, however, violate the time window constraints associated with the ships and the berths, and the QC availability. The time window constraint on ship $i$ on a berth $k$ is violated if the arrival time $T_{i}^{k}$ of the ship is larger than the time window's upper bound $b_{i}$. Berthing before $a_{i}$ is not allowed; in other words, $T_{i}^{k} \geq a_{i}$. Similarly, the time window of berth $k$ is violated when the completion time of a ship $i$ assigned to berth $k$ is larger than the berth time window's upper bound $b^{k}$.

Let $c(s)$ denote the cost of solution defined in (30), and let $w_{1}(s)$ denote the total violation of ship's time window constraints, equal to the sum of the violations on the $n$ ships. We indicate as $w_{2}(s)$ the total violation of berth's time window constraints, equal to the sum of the violations on the $\mathrm{m}$ berths. Finally, let $w_{3}(\mathrm{~s})$ be the total violation of $\mathrm{QC}$ availability for each time step of the planning horizon. Solutions are then evaluated by means of a penalized cost function $f(s)=c(s)+\alpha_{1} w_{1}(s)+\alpha_{2} w_{2}(s)+\alpha_{3} w_{3}(s)$, where the $\alpha$ values are positive parameters. By dynamically adjusting the value of these parameters, the relaxation mechanism facilitates the exploration of the search space and is particularly useful for tightly constrained instances.

The tabu search method is based on the definition of attributes used to characterize the solutions of $S$. They are also used to control tabu tenures and to implement a diversification strategy. An attribute set $B(s)=\{(i, k)$ : ship $i$ is assigned to berth $k\}$ is associated to each solution $s \in S$. The neighborhood $\mathrm{N}(\mathrm{s})$ of a solution $\mathrm{s}$ is defined by applying a simple operator that removes an attribute $(i, k)$ from $B(s)$ and replaces it with another attribute $\left(i, k^{\prime}\right)$, where $k \neq k^{\prime}$. When ship $i$ is removed from berth $k$, the sequence 
is simply reconnected by linking the predecessor and successor of the ship. Insertion in sequence $k^{\prime}$ is then performed between two consecutive ships so as to minimize the value of $f(s)$. When a ship $i$ is removed from berth $k$, its reinsertion in that berth is forbidden for the next $\theta$ iterations by assigning a tabu status to the attribute $(i, k)$.

An aspiration criterion allows the revocation of the tabu status of an attribute if that would allow the search process to reach a solution of smaller cost than that of the best solution identified having that attribute. To diversify the search, any solution $\bar{s} \in N(s)$ such that $f(\bar{s}) \geq f(s)$ is penalized by a factor proportional to the addition frequency of its attributes, and by a scaling factor. More precisely, let $\xi_{i k}$ be the number of times attribute $(i, k)$ has been added to the solution during the process and let $\zeta$ be the number of the current iteration. A penalty $p(\bar{s})=\beta c(\bar{s}) \xi_{i k} / \zeta$ is added to $f(\bar{s})$. The scaling factor $c(\bar{s})$ introduces a correction to adjust the penalties with respect to the total solution cost. Finally, the parameter $\beta$ is used to control the intensity of the diversification. These penalties have the effect of driving the search process toward less explored regions of the search space. For notational convenience, assume that $p(\bar{s})=0$ if $f(\bar{s})<f(s)$.

In order to to generate a starting solution, the algorithm assigns the ships to the berths at random. This initial solution is constructed by relaxing the time window and QC availability constraints, and therefore it is usually infeasible. However, this is not an issue for the tabu search heuristics.

The search starts from this initial solution and selects, at each iteration, the best non-tabu solution $\bar{s} \in \mathrm{N}(\mathrm{s})$. After each iteration, the value of parameters $\alpha_{1}, \alpha_{2}$, and $\alpha_{3}$ are modified by a factor $1+\delta$, where $\delta>$ 0 . For example, if the current solution is feasible with respect to ship's time window constraints, the value of $\alpha_{1}$ is divided by $1+\delta$; otherwise, it is multiplied by $1+\delta$. Analogously for the berth's time window and QC availability constraints, i.e. parameters $\alpha_{2}$ and $\alpha_{3}$, respectively. This process is repeated for $\eta$ iterations and the best feasible solution $s^{*}$ is updated throughout the search. 


\subsection{Profile update via mathematical programming}

The profiles' updating procedure relies on the MILP formulation for TBAP illustrated in Section 3.4. The basic idea of this step is to use the information of reduced costs in order to be able to update vector $\lambda$ of QC profiles' assignment in a smart way.

Let $s^{*}=[\bar{x}, \bar{y}, \bar{T}]$ be the BAP solution provided by tabu search for a given $\mathrm{QC}$ profile assignment $\bar{\lambda}$. in particular, we are interested in reduced costs of variables $\lambda$, which we denote $\tilde{\lambda}$. We remark that a BAP solution plus a QC assignment represent a feasible solution for TBAP. At each iteration, we solve the linear relaxation of the MILP formulation, with the additional constraints:

$$
\begin{aligned}
& \bar{x}-\epsilon \leq x \leq \bar{x}+\epsilon \\
& \bar{y}-\epsilon \leq y \leq \bar{y}+\epsilon \\
& \overline{\mathrm{T}}-\epsilon \leq \mathrm{T} \leq \overline{\mathrm{T}}+\epsilon \\
& \bar{\lambda}-\epsilon \leq \lambda \leq \bar{\lambda}+\epsilon
\end{aligned}
$$

As remarked, e.g., by Desrosiers and Lübbecke (2005), the shadow prices of constraints (31)-(34) are the reduced costs of original variables $x, y, T$ and $\lambda$. We therefore identify the $\lambda_{i}^{p}$ variable with the maximum reduced cost and we assign this new profile $p$ to ship $i$. If all reduced costs are non-positive or other stopping criteria as maximum number of iterations or time limit are reached, the procedure terminates.

A tabu mechanism has been implemented in order to prevent insisting on the same ship; the length of this tabu list has been fixed to $0.5 n \times \bar{p}$.

It may happen that the tabu search returns a BAP solution which is infeasible for TBAP with respect to time windows and/or the QC availability. In this case the profiles' update via mathematical programming cannot be performed. We therefore update the set of profiles by randomly assigning a new QC profile to each ship. 


\section{Computational results}

The heuristics has been implemented in C++ using GLPK 4.31 and tested on the same set of instances illustrated in Section 4.

Experiments have been run for $n \times \bar{p}$ iterations and a time limit of 1 hour for classes $10 \times 3,20 \times 5,30 \times 5$ and 3 hours for classes $40 \times 5,50 \times 8$, $60 \times 13$. The initialization assigns the best value profile to each ship. Ties are broken arbitrarily. The internal tabu search has a maximum of $\eta=30 \times n$ iterations, and the other parameters are set as follows:

- $\theta$ : tabu duration equal to $\lfloor 7.5 \log n\rfloor$;

- $\beta$ : diversification intensity parameter equal to $0.015 \sqrt{\mathrm{nm}}$;

- $\delta$ : penalty adjustment parameter equal to 2 .

Results are compared to the best solution found by CPLEX for either the MILP or MIQP formulation and illustrated in Tables 4, 5 and 6, The heuristics is able to find feasible solutions in 70 out of 72 instances, whereas CPLEX succeeds at that only on 20 instances, the smaller ones. The two instances where the heuristics fails at finding a feasible solution are characterized by an high number of profiles per vessel $(\bar{p}=30)$. We observe that with a lower number of profiles per vessel $(\bar{p}=10$, and $\bar{p}=20)$ the heuristics always succeeds in reaching feasibility. Furthermore, our algorithm is up to 2 order of magnitude faster, especially on small instances.

Class $10 \mathrm{x} 3$ is the only one where CPLEX performs slightly better than the heuristics, with an average of 99.00 and 98.59, respectively, and 3 optimums found by CPLEX. However, the heuristics is much faster, solving the problem in less than 30 seconds against the time limit of 1 hour set for CPLEX.

Class $20 \times 5$ is always solved by the heuristics in less then 5 minutes, with an average quality of the solution of 97.29, while CPLEX only solves 4 instances out of 12 , in 2 hours, with lower quality (95.37 on average).

Remarkably, our heuristics performs very well also on the instances of bigger size, where CPLEX generally fails. For the solved instances the quality of the solutions is always greater than 94.11 (instance 60x13:H2_20), with an average value of 96.06 . 
$10 \times 3$

$20 \times 5$

\begin{tabular}{lccc|lccc}
\hline Instance & CPLEX & HEUR & Time (sec) & Instance & CPLEX & HEUR & Time (sec) \\
\hline H1_10 & 99.17 & 98.52 & 7 & H1_10 & - & 97.26 & 81 \\
H1_20 & 97.96 & 98.36 & 15 & H1_20 & 94.33 & 97.19 & 172 \\
H1_30 & 98.76 & 98.33 & 27 & H1_30 & 93.74 & 97.37 & 259 \\
\hline H2_10 & 99.26 & 98.92 & 7 & H2_10 & - & 97.27 & 82 \\
H2_20 & 96.97 & 98.48 & 16 & H2_20 & 96.66 & 97.38 & 173 \\
H2_30 & 96.79 & 98.17 & 28 & H2_30 & - & 97.26 & 274 \\
\hline L1_10 & 100.00 & 99.12 & 6 & L1_10 & - & 97.30 & 74 \\
L1_20 & 100.00 & 99.01 & 15 & L1_20 & - & 97.25 & 158 \\
L1_30 & 99.99 & 98.29 & 26 & L1_30 & - & 97.06 & 254 \\
\hline L2_10 & 99.63 & 98.92 & 6 & L2_10 & - & 97.55 & 80 \\
L2_20 & 99.42 & 98.68 & 15 & L2_20 & 96.74 & 97.39 & 170 \\
L2_30 & 100.00 & 98.22 & 27 & L2_30 & - & 97.25 & 295 \\
\hline
\end{tabular}

Table 4: Heuristics computational results on classes $10 x 3$ and $20 x 5$.

\begin{tabular}{lccc|lccc}
\multicolumn{9}{c}{ 30x5 } \\
\hline Instance & CPLEX & HEUR & Time $(\mathrm{sec})$ & Instance & CPLEX & HEUR & Time (sec) \\
\hline H1_10 & - & 95.67 & 340 & H1_10 & - & 97.38 & 1104 \\
H1_20 & - & 95.31 & 677 & H1_20 & - & 97.38 & 2234 \\
H1_30 & - & 95.54 & 1009 & H1_30 & - & 97.25 & 3387 \\
\hline H2_10 & - & 95.88 & 316 & H2_10 & - & 97.40 & 1095 \\
H2_20 & - & 95.81 & 684 & H2_20 & - & 97.33 & 2198 \\
H2_30 & - & 95.30 & 969 & H2_30 & - & 97.27 & 3296 \\
\hline L1_10 & - & 96.55 & 324 & L1_10 & 94.92 & 97.41 & 1421 \\
L1_20 & - & 96.43 & 652 & L1_20 & 94.47 & 97.14 & 2996 \\
L1_30 & - & 96.18 & 966 & L1_30 & - & 96.20 & 4862 \\
\hline L2_10 & - & 95.68 & 308 & L2_10 & - & 97.41 & 1382 \\
L2_20 & - & 95.12 & 614 & L2_20 & 94.93 & 97.34 & 3144 \\
L2_30 & - & - & 920 & L2_30 & 94.61 & 96.60 & 4352 \\
\hline
\end{tabular}

Table 5: Heuristics computational results on classes $30 x 5$ and $40 x 5$. 


\begin{tabular}{lccc|lccc}
\multicolumn{9}{c}{$\mathbf{5 0 x 8}$} \\
\hline Instance & CPLEX & HEUR & Time $(\mathrm{sec})$ & Instance & CPLEX & HEUR & Time (sec) \\
\hline H1_10 & - & 96.52 & 3291 & H1_10 & - & 95.40 & 6332 \\
H1_20 & - & 96.37 & 6020 & H1_20 & - & 95.07 & 10809 \\
H1_30 & - & 96.21 & 9432 & H1_30 & - & 94.76 & 10807 \\
\hline H2_10 & - & 96.03 & 3066 & H2_10 & - & 95.54 & 6397 \\
H2_20 & - & 95.64 & 6180 & H2_20 & - & 94.11 & 10803 \\
H2_30 & - & 95.16 & 9501 & H2_30 & - & - & 10806 \\
\hline L1_10 & - & 95.97 & 2752 & L1_10 & - & 95.67 & 5807 \\
L1_20 & - & 96.04 & 6467 & L1_20 & - & 95.40 & 10803 \\
L1_30 & - & 95.80 & 9119 & L1_30 & - & 94.45 & 10806 \\
\hline L2_10 & - & 96.18 & 3157 & L2_10 & - & 95.63 & 5986 \\
L2_20 & - & 95.96 & 5857 & L2_20 & - & 95.64 & 10809 \\
L2_30 & - & 96.27 & 8783 & L2_30 & - & 95.34 & 10804 \\
\hline
\end{tabular}

Table 6: Heuristics computational results on classes $50 x 8$ and $60 x 13$.

\section{Conclusions and future work}

We have studied the integration, at the tactical level, of the berth allocation problem with the assignment of quay cranes from the point of view of a container terminal, in the context of a negotiation process with shipping lines. We have characterized this new decision problem and illustrated the concept of QCs assignment profiles. Two mixed integer programming formulations have been presented, with a quadratic and a linearized objective function respectively. Both models have been validated on instances based on real data using a commercial solver. These tests show that the problem is hardly solvable already on small instances; hence we have tackled the computational complexity of TBAP by devising a bi-level heuristic algorithm able to provide good feasible solutions in a reasonable amount of time.

As a next step, we are interested in obtaining good upper bounds on the optimal solution. Decomposition methods seem to be a promising way to face the problem. In fact, we are considering a reformulation based on 
Dantzig-Wolfe decomposition and column generation, and an incremental approach based on Lagrangian dual, in order to exploit the structure of TBAP and its relation with the BAP formulation, aiming at saving computational time by solving subproblems via inexact or truncated methods.

With respect to the application, we remark that the main contribution is represented by the simultaneous control of the terminal on critical resources such as berths and quay cranes, in addition to the added value given by the integration, in a more direct way, of different terminal's costs.

\section{Acknowledgments}

We would like to thank Carmine Crudo and Vincenzo Perri at Medcenter Container Terminal S.p.a. in Gioia Tauro (Italy) for many helpful discussions on the subject. The work of G. Giallombardo and L. Moccia has been partially supported by the Italian Ministry of University through the research grant "LOGNET - PROMIS: Logistic Process Management and Intelligence System."

\section{References}

Cordeau, J. F., Gaudioso, M., Laporte, G. and Moccia, L. (2006). A memetic heuristic for the generalized quadratic assignment problem, INFORMS Journal on Computing 18(4): 433-443.

Cordeau, J. F., Gaudioso, M., Laporte, G. and Moccia, L. (2007). The service allocation problem at the Gioia Tauro maritime terminal, European Journal of Operational Research 176: 1167-1184.

Cordeau, J. F., Laporte, G., Legato, P. and Moccia, L. (2005). Models and tabu search heuristics for the berth-allocation problem, Transportation Science 39: 526-538.

Crainic, T. G. and Kim, K. H. (2007). Intermodal transportation, in C. Barnhart and G. Laporte (eds), Transportation, Vol. 14 of Hand- 
books in Operations Research and Management Science, Elsevier, pp. 467-537.

Dai, J., Lin, W., Moorthy, R. and Teo, C. P. (2007). Berth allocation planning optimization in container terminals, in C. S. Tang, C. P. Teo and K. K. Wei (eds), Supply Chain Analysis, Vol. 119 of International Series in Operations Research \& Management Science, Springer, pp. 69-104.

Desrosiers, J. and Lübbecke, M. E. (2005). A primer in column generation, in G. Desaulniers, J. Desrosiers and M. Solomon (eds), Column Generation, GERAD, chapter 1, pp. 1-32.

Hahn, P., Kim, B. J., Guignard, M., Smith, J. and Zhu, Y. R. (2008). An algorithm for the generalized quadratic assignment problem, Computational Optimization and Applications 40(3): 351-372.

Imai, A., Chen, H. C., Nishimura, E. and Papadimitriou, S. (2008). The simultaneous berth and quay crane allocation problem, Transportation Research Part E 44: 900-920.

Imai, A., Nagaiwa, K. and Chan, W. T. (1997). Efficient planning of berth allocation for container terminals in Asia, Journal of Advanced Transportation 31: 75-94.

Imai, A., Nishimura, E., Hattori, M. and Papadimitriou, S. (2007). Berth allocation at indented berths for mega-containerships, European Journal of Operational Research 179: 579-593.

Imai, A., Nishimura, E. and Papadimitriou, S. (2001). The dynamic berth allocation problem for a container port, Transportation Research Part B 35: 401-417.

Imai, A., Nishimura, E. and Papadimitriou, S. (2003). Berth allocation with service priority, Transportation Research Part B 37: 437-457.

Imai, A., Sun, X., Nishimura, E. and Papadimitriou, S. (2005). Berth allocation in a container port: using a continuous location space approach, Transportation Research Part B 39: 199-221. 
Kim, K. H. and Park, Y. M. (2004). A crane scheduling method for port container terminals, European Journal of Operational Research 156: 752-768.

Lim, A. (1998). The berth planning problem, Operations Research Letters 22: $105-110$.

Meisel, F. and Bierwirth, C. (2006). Integration of berth allocation and crane assignment to improve the resource utilization at a seaport container terminal, Operations Research Proceedings 2005, Springer, pp. 105-110.

Meisel, F. and Bierwirth, C. (2009). Heuristics for the integration of crane productivity in the berth allocation problem, Transportation Research Part E 45(1): 196-209.

Moccia, L., Cordeau, J. F., Gaudioso, M. and Laporte, G. (2006). A branchand-cut algorithm for the quay crane scheduling problem in a container terminal, Naval Research Logistics 53: 45-59.

Moccia, L., Cordeau, J.-F., Monaco, M. F. and Sammarra, M. (2009). A column generation heuristic for a dynamic generalized assignment problem, Computers \& Operations Research doi:10.1016/j.cor.2008.11.022(36): $2670-2681$.

Monaco, M. F., Moccia, L. and Sammarra, M. (2009). Operations research for the management of a transhipment container terminal. The Gioia Tauro case, Maritime Economics \& Logistics 11: 7-35.

Monaco, M. F. and Sammarra, M. (2007). The berth allocation problem: a strong formulation solved by a lagrangean approach, Transportation Science 41: 265-280.

Moorthy, R. and Teo, C. P. (2006). Berth management in container terminal: the template design problem, OR Spectrum 28: 495-518.

Nishimura, E., Imai, A. and Papadimitriou, S. (2001). Berth allocation planning in the public berth system by genetic algorithms, European Journal of Operational Research 131: 282-292. 
Park, Y. M. and Kim, K. H. (2003). A scheduling method for berth and quay cranes, OR Spectrum 25: 1-23.

Stahlbock, R. and Voss, S. (2008). Operations research at container terminals: a literature update, $O R$ Spectrum 30: 1-52.

Steenken, D., Voss, S. and Stahlbock, R. (2004). Container terminal operation and operations research - a classification and literature review, OR Spectrum 26: 3-49.

UNCTAD (2008). Review of maritime transport, Technical report, United Nations, New York and Geneva.

Vis, I. F. A. and de Koster, R. (2003). Transshipment of containers at a container terminal: An overview, European Journal of Operational Research 147: 1-16.

Wang, F. and Lim, A. (2007). A stochastic beam search for the berth allocation problem, Decision Support Systems 42: 2186-2196. 